\title{
PROPERTIES OF THE DIFFUSE X-RAY BACKGROUND TOWARD MBM20 WITH SUZAKU
}

\author{
A. Gupta ${ }^{1}$, M. Galeazzi ${ }^{1}$, D. Koutroumpa ${ }^{2}$, R. Smith $^{3}$, and R. Lallement ${ }^{4}$ \\ ${ }^{1}$ Physics Department, University of Miami, Coral Gables, FL 33124, USA; galeazzi@physiscs.miami.edu \\ 2 NASA/GSFC, Code 662, Greenbelt, MD 20771, USA \\ ${ }^{3}$ Harvard Smithsonian Center for Astrophysics, Cambridge, MA 02138, USA \\ ${ }^{4}$ CNRS Service d'Aéronomie, FR 91371 Verrieres Buisson, France \\ Received 2009 September 13; accepted 2009 October 22; published 2009 November 24
}

\begin{abstract}
We used Suzaku observations of the molecular cloud MBM20 and a low neutral hydrogen column density region nearby to separate and characterize the foreground and background diffuse X-ray emission. A comparison with a previous observation of the same regions with XMM-Newton indicates a significant change in the foreground flux which is attributed to Solar Wind Charge eXchange (SWCX). The data have also been compared with previous results from similar "shadow" experiments and with a SWCX model to characterize its O VII and O vIII emission.
\end{abstract}

Key words: X-rays: diffuse background

\section{INTRODUCTION}

Our current interpretation of the diffuse X-ray emission below $1 \mathrm{keV}$ includes a combination of five components: Solar Wind Charge eXchange (SWCX), Local Bubble (LB), Galactic Halo (GH), Warm-Hot Intergalactic Medium (WHIM), and unresolved point sources (e.g., Gupta \& Galeazzi 2009; Galeazzi et al. 2009). Resolving the different components is made particularly difficult due to the similar spectra of the components, primarily X-ray lines from heavily ionized metals. Compounding the problem, these lines are poorly resolved by the CCD cameras onboard current X-ray satellites.

Shadowing observations offer a tool to simplify the study of the various components by separating local (less than about $100 \mathrm{pc}$ ) and non-local components. A typical shadow experiment consists of two observations, one in the direction of a high latitude, high neutral hydrogen density cloud at a distance of 50$200 \mathrm{pc}$, the other toward a low neutral hydrogen column density sightline as close as possible to the cloud. As the cloud absorbs most of the background X-ray emission, a comparison of the two observations separates the foreground (LB plus SWCX) and background (GH, WHIM, and unresolved point sources) emission. Simultaneous spectral analysis of the two observations then determines the physical parameters of the different components.

The results, however, are complicated by the properties of the SWCX component, which varies both in spectra composition and flux on a scale of hours to days. The SWCX component originates in the interaction of the highly ionized solar wind with neutral gas in the Earth's atmosphere and in the interplanetary medium. The charge exchange occurs when an electron jumps from the neutral atom to an excited level in the ion. The electron then cascades to the lower energy level of the ion, emitting soft $\mathrm{X}$-rays and other lines in the process. While the properties of the SWCX emission are not fully understood, it seems quite clear that, for a proper comparison of on-cloud and off-cloud data, the two observations must be temporally as close as possible. Even in such conditions, however, a careful monitoring and simulation of the expected SWCX emission is needed.

We have obtained spectra of the Soft X-Ray Background (SXRB) in the direction of the high latitude, neutral hydrogen cloud MBM20 and a low neutral hydrogen column density region nearby that we called the Eridanus Hole $(\mathrm{EH})$ using the X-ray Imaging Spectrometer (XIS; Koyama et al. 2007) onboard the Suzaku X-ray observatory. The XIS is an excellent tool for studying the SXRB, due to its low and stable non$\mathrm{X}$-ray background and good spectral resolution. The targets are identical to those observed in Galeazzi et al. (2007) using XMMNewton.

To build a consistent picture of the diffuse X-ray background we compared the Suzaku observation with the previous $X M M-N e w t o n$ result and with similar shadow observations in the direction of the high latitude molecular cloud MBM12 (Smith et al. 2005, 2006) and a filament in the southern Galactic hemisphere (Henley et al. 2007; Henley \& Shelton 2008). We also used the model recently developed by Koutroumpa et al. (2007) to estimate the emission from SWCX. The model is time dependent and includes factors such as solar cycle phase, the observation position, and the line of sight (LOS).

The data reduction is discussed in Section 2 and the analysis and X-ray results in Section 3. Section 4 compares our result with the previous XMM-Newton observation and with other recent shadow experiments, and Section 5 to the characterization of SWCX, including models of the SWCX emission during the Suzaku and XMM-Newton observations.

\section{OBSERVATIONS OF MBM20 AND ERIDANUS HOLE}

MBM20 and the EH were observed with Suzaku in 2008 February and 2007 July, respectively. Note that the temporal gap between the observations is large compared to the typical time variation of the SWCX and will be discussed in Section 5. The details of the observations are reported in Table 1. MBM20 is a high-density, high-latitude star-forming cloud located at or within the edge of the LB (Galeazzi et al. 2007). Its mass is $84 M_{\odot}$ and it is located at coordinates $l=211^{\circ} 23^{\prime} 53^{\prime \prime} .2$, $b=-36^{\circ} 32^{\prime} 41^{\prime \prime} .8$, southwest of the Orion star forming complex. Based on interstellar NaI D absorption lines the distance of MBM20 is evaluated between $112 \pm 15 \mathrm{pc}$ and $161 \pm 21 \mathrm{pc}$ (Hearty et al. 2000). The EH, at coordinates $l=213^{\circ} 25^{\prime} 52^{\prime \prime} .3$, $b=-39^{\circ} 5^{\prime} 26^{\prime \prime} 6$, is a region of low neutral hydrogen column density located about 2 deg from the highest-density part of MBM20 (Figure 1).

\subsection{Data Reduction}

We used the Suzaku data reprocessed to version 2.0 and the analysis was performed with HEAsoft ${ }^{5}$ version 6.4 and

\footnotetext{
5 See http://heasarc.nasa.gov/lheasoft/.
} 
Table 1

Details of Our Suzaku Observations

\begin{tabular}{lcccc}
\hline \hline \multicolumn{1}{c}{ Target } & Observation ID & Start Time (UT) & End Time (UT) & Exposure (ks) \\
\hline MBM20 & 502075010 & 2008 Feb 11 14:41:19 & 2008 Feb 14 16:45:11 & 69.2 \\
Eridanus Hole & 502076010 & 2007 Jul 30 00:51:47 & 2007 Aug 01 05:11:19 & 84.6 \\
\hline
\end{tabular}

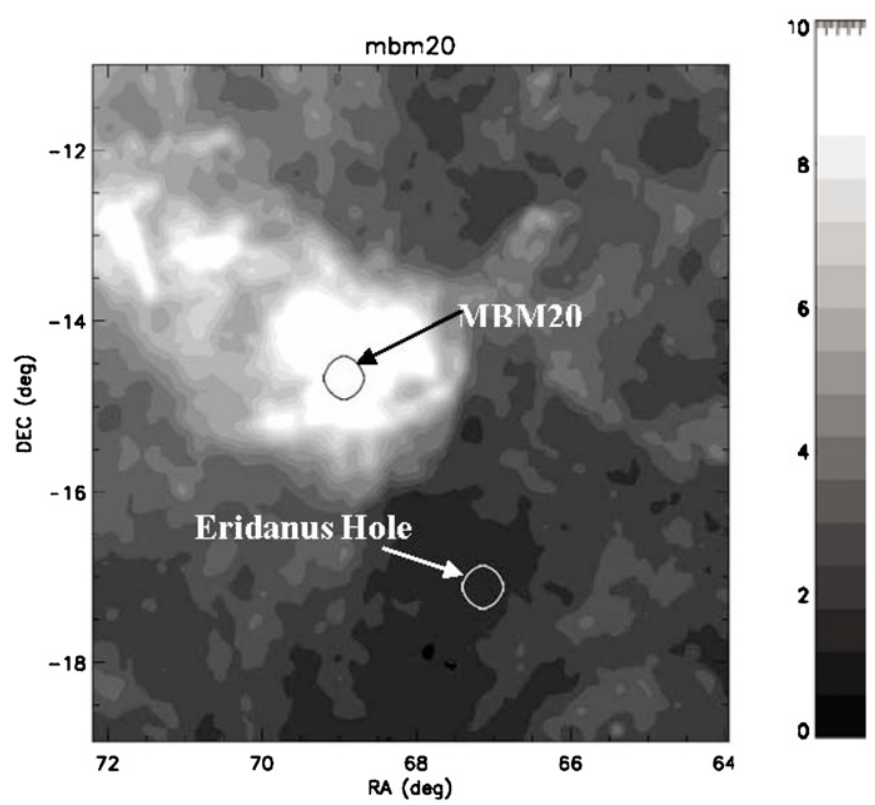

Figure 1. IRAS $100 \mu \mathrm{m}$ map of MBM20 and surroundings showing the two pointing used in this investigation. The units are $\mathrm{MJy} \mathrm{sr}^{-1}$.

XSPEC 12.4.0. We started the event screening from the cleaned event file, in which the selection of the event grade and bad CCD column, and the removal of hot and flickering pixels by the "cleansis" ftool, were already conducted (Suzaku Data Reduction Guide ${ }^{6}$ ).

In our analysis, we use only data from the XIS1 detector, as this has the greatest sensitivity at low energies. We combined the data taken in the $3 \times 3$ and $5 \times 5$ observation mode. For that, first we convert the $5 \times 5$ mode data to $3 \times 3$ mode data using Ftool "xis $5 \times 5$ to $3 \times 3$," then merged both files with the help of Ftool "ftmerge." The cleaned event files are by default filtered to exclude times within 436 s of Suzaku passing through

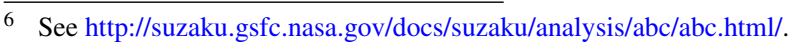

the South Atlantic Anomaly (SAA), and when Suzaku's LOS is elevated above the Earths limb by less than $5^{\circ}$, or is less than $20^{\circ}$ from the bright-Earth terminator. We decided to expand this to exclude events with Earth-limb elevation angle less than $10^{\circ}$, as there are some excess events in the $0.5-0.6 \mathrm{keV}$ band in the $5^{\circ}-10^{\circ}$ range (Smith et al. 2006).

Due to Suzaku's broad point-spread function (half-power diameter $\sim 2^{\prime}$; Mitsuda et al. 2007), it is hard to detect point sources. Therefore, to remove sources which could contaminate our SXRB spectra, we used the location of sources determined in XMM-Newton observations (see Figure 2). We extracted spectra from the full XIS1 field of view, after removing abovementioned point sources and the corners of the detectors which contained the onboard Fe-55 calibration sources.

\subsection{Background Removal}

Suzaku is in a low-Earth orbit, so it is significantly shielded from the particle background that strongly affects XMM-Newton and Chandra. The effectiveness of this shielding is dependent upon the "cut-off rigidity" (COR) of the Earth's magnetic field, which varies as Suzaku traverses its orbit. During times with larger COR values, fewer particles are able to penetrate to the satellite and to the XIS detectors. We excluded times when the COR was less than $8 \mathrm{GV}$, which is higher than the default value (COR $4 \mathrm{GV}$ ) for both observations, as the lowest background was desired.

Although it is reduced by the Earth's magnetic field, Suzaku still has a noticeable particle background. We can estimate the appropriate particle background from a database of the night Earth data (NXB). NXB was collected when the telescope was pointed at the night Earth (elevation less than $-5^{\circ}$, and pointed at night side rather than day). The event files in the database have been carefully screened for telemetry saturation and other artifacts. We constructed the spectra of the night earth data using Ftool "xisnxbgen" (Tawa et al. 2008), which sorts the NXB data by COR values, generates an NXB spectrum and image for each COR range, and combines them weighted by exposure time ratio of each COR range during GTIs in our
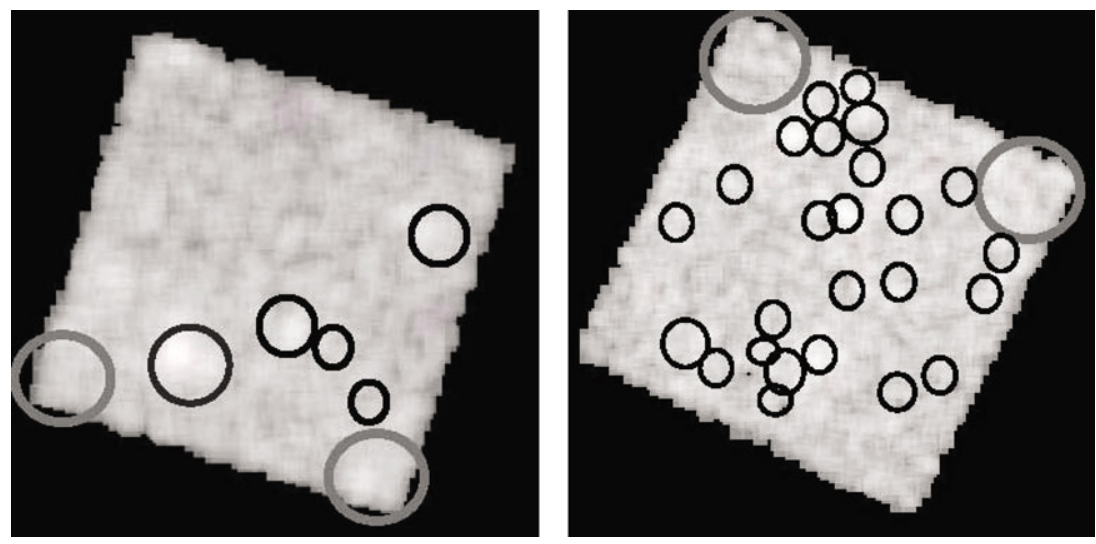

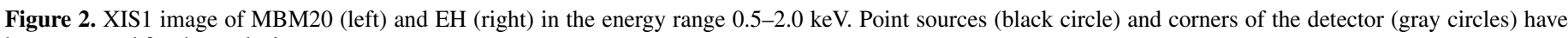
been removed for the analysis. 
Table 2

Model Parameters of the Spectral Fits

\begin{tabular}{|c|c|c|c|c|c|c|c|}
\hline \multirow[t]{2}{*}{ Data set } & \multicolumn{2}{|c|}{ Local Component } & \multicolumn{2}{|c|}{ Galactic Halo } & \multicolumn{2}{|c|}{ Power Law } & \multirow[t]{2}{*}{$\chi^{2} / d o f$} \\
\hline & $\begin{array}{c}T \\
\left(10^{6} \mathrm{~K}\right) / \mathrm{keV}\end{array}$ & $\begin{array}{c}\mathrm{EM}^{\mathrm{a}} \\
\left(\mathrm{cm}^{-6} \mathrm{pc}\right)\end{array}$ & $\begin{array}{c}T \\
\left(10^{6} \mathrm{~K}\right) / \mathrm{keV}\end{array}$ & $\begin{array}{c}\mathrm{EM} \\
\left(\mathrm{cm}^{-6} \mathrm{pc}\right)\end{array}$ & $\Gamma^{\mathrm{b}}$ & Norm $^{\mathrm{c}}$ & \\
\hline MBM20 & $0.78 / 0.067$ & 0.041 & $1.87 / 0.16$ & 0.0034 & 1.48 & 8.4 & $167.6 / 151$ \\
\hline $\mathrm{EH}$ & $1.24 / 0.106$ & 0.007 & $2.46 / 0.21$ & 0.0016 & 1.57 & 8.1 & $134.3 / 151$ \\
\hline$(\mathrm{MBM} 20+\mathrm{EH})^{\mathrm{d}}$ & $0.83 / 0.071$ & 0.027 & $2.11 / 0.18$ & 0.0027 & 1.53 & 8.3 & $304.9 / 308$ \\
\hline$(\mathrm{MBM} 20+\mathrm{EH})^{\mathrm{e}}$ & $0.76 / 0.067$ & 0.056 & $2.12 / 0.19$ & 0.0031 & 1.33 & 9.1 & $306.2 / 352$ \\
\hline$(\mathrm{MBM} 20+\mathrm{EH})^{\mathrm{f}}$ & $1.12 / 0.096$ & 0.0078 & $2.23 / 0.19$ & 0.0035 & 2.2 & 14.8 & $768 / 640$ \\
\hline
\end{tabular}

Notes.

a Emission measure.

${ }^{b}$ Index of absorbed power-law fit.

${ }^{c}$ Normalization of power-law fit at $1 \mathrm{keV}$ in units of photons $\mathrm{keV}^{-1} \mathrm{~s}^{-1} \mathrm{~cm}^{-2} \mathrm{sr}^{-1}$.

d Suzaku data only.

e Suzaku and RASS data.

${ }^{f}$ XMM-Newton result from Galeazzi et al. (2007).

spectral data file. The background spectra were then subtracted from the corresponding source spectra.

\subsection{XIS Response}

We calculated the XIS detector effective area using the tool "xissimarfgen" (Ishisaki et al. 2007). This tool takes into account the spatially varying contamination on the optical blocking filters of the XIS sensors which reduces the detector efficiency at low energies (Koyama et al. 2007). For the ancillary response file (ARF) calculations we assumed a uniform source of radius $20^{\prime}$ and used a detector mask which removed the bad pixel regions. To generate the redistribution matrix file (RMF), we used the ftool "xisrmfgen."

\section{ANALYSIS}

We first fit a model to our spectra consisting of three components: a LB component, modeled as an unabsorbed plasma with thermal emission in collisional ionization equilibrium (CIE); a hotter $\mathrm{GH}$ emission, modeled as an equilibrium thermal plasma component absorbed by the gas in the Galactic disk; and an unresolved extragalactic source component, modeled with an absorbed power law. This is the same model used in Galeazzi et al. (2007). As extensively discussed in Sections 4 and 5, the data are affected by "contamination" due to SWCX which limits the significance of the results obtained with this model. However, with the limited energy resolution of the CCD detectors the mentioned model works quite well and allows for a straightforward comparison with previous results.

We used the XSPEC version 12.4 (Arnaud \& Dormer 2002) to fit both spectra, in the energy range $0.4-5.5 \mathrm{keV}$. For plasma thermal emission, the Astrophysical Plasma Emission Code (APEC) was used (Smith \& Cox 2001), and for the absorption, we used the XSPEC wabs model, which uses cross-sections from Wisconsin (Morrison \& McCammon 1983) and uses the Anders \& Ebihara (1982) relative abundances. We fit the abovementioned model to the Suzaku spectra of MBM20 and the EH. As in Galeazzi et al. (2007), we used the IRAS $100 \mu \mathrm{m}$ maps to evaluate the neutral hydrogen density in the two regions. The IRAS average brightness is $13.34 \mathrm{MJy} \mathrm{sr}^{-1}$, and $0.73 \mathrm{MJy} \mathrm{sr}^{-1}$ for MBM20, and the EH, respectively. Using the "typical" high-latitude $100 \mu \mathrm{m} / \mathrm{NH}$ ratio of $0.85 \times 10^{-20} \mathrm{~cm}^{2} \mathrm{MJy} \mathrm{sr}^{-1}$ (Boulanger \& Perault 1988) the estimated neutral hydrogen densities are $1.59 \times 10^{21} \mathrm{~cm}^{-2}$ and $0.86 \times 10^{20} \mathrm{~cm}^{-2}$ respectively.
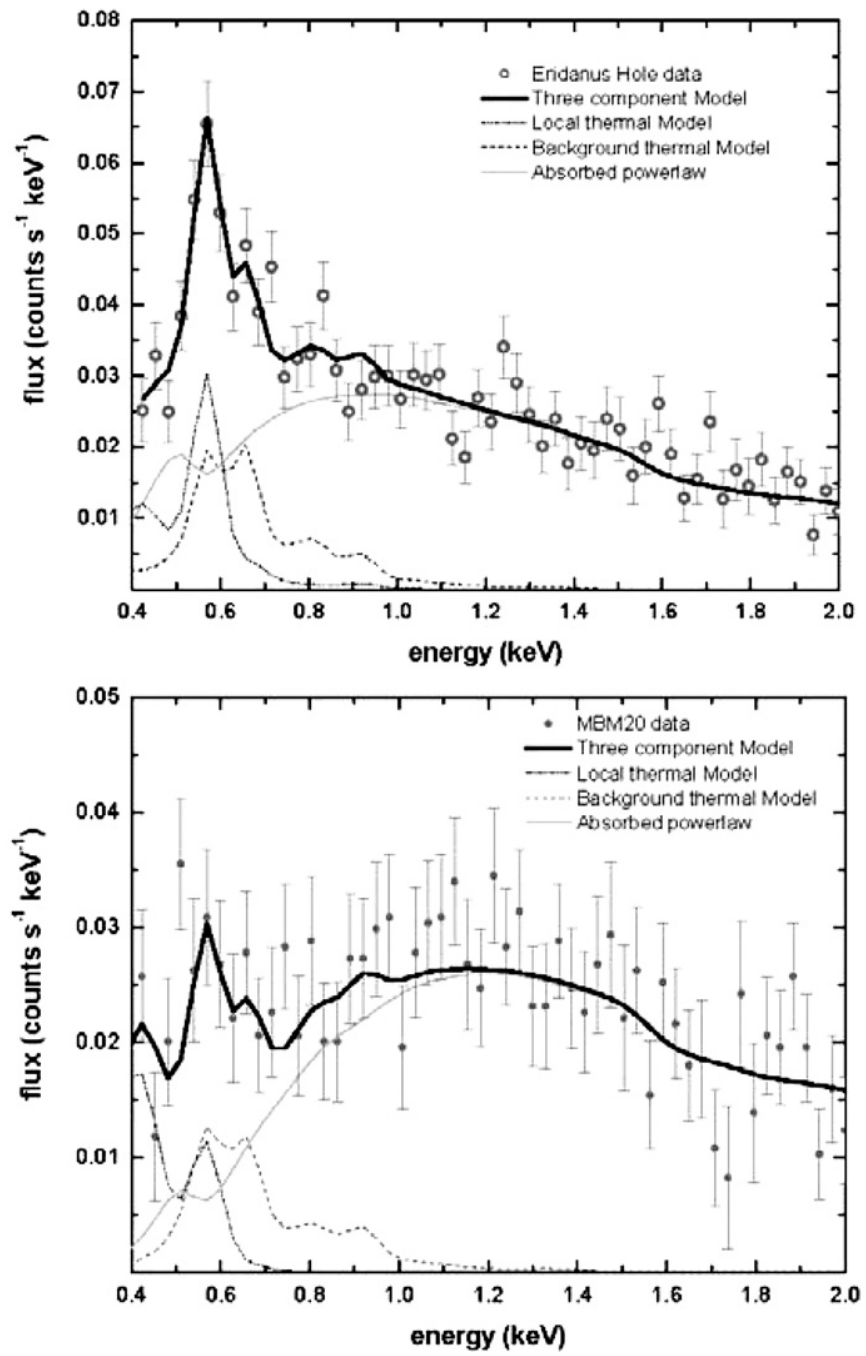

Figure 3. EH (top) and MBM20 (bottom) Suzaku spectra, with the best fitting three-component model.

The fits are shown in Figure 3, along with the best-fitting multicomponent spectral model. The model parameters are reported in Table 2.

We also tried to fit the above-mentioned model simultaneously to our MBM20 and EH Suzaku spectra with a single set 


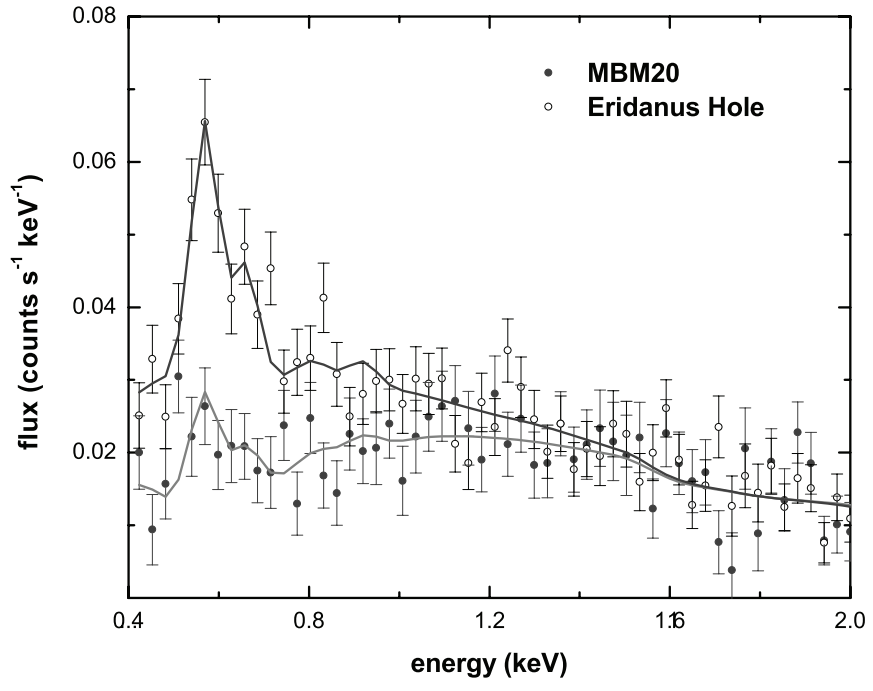

Figure 4. Simultaneous fit for MBM20 and EH Suzaku data.

of parameters, except for the neutral hydrogen column density. The fits are shown in Figure 4, and the model parameters are presented in Table 2.

To extend the analysis further, we also included data from the ROSAT All-Sky Survey (RASS) in the same directions. We extracted RASS data in the ROSAT bands R1-R7 (Snowden et al. 1998) and scaled them to the same field of view as our Suzaku data sets for both MBM20 and the EH. We then performed a global fit of the four data sets simultaneously with a single set of parameters. The fit results are reported in Table 2, and the data are shown in Figure 5. Overall, the model gives a good fit to the data (reduced $\chi^{2}=0.87$ for 352 degrees of freedom), however, the fit to some of the ROSAT bands is rather poor.

We used our fit results to obtain O VII and O VIII intensities, since at temperatures of million Kelvins, $\mathrm{O}$ VII and $\mathrm{O}$ VIII lines are the dominant features. In our Suzaku spectra of MBM20 and $\mathrm{EH}$, the blended $\mathrm{O}$ VII triplet at 561,569 and $574 \mathrm{eV}$ is clearly visible in both observations, while the O vIII line at $654 \mathrm{eV}$ is barely visible in the MBM20 data set and lies within the statistical uncertainty in the EH data set. The $\mathrm{O}$ VII and $\mathrm{O}$ VIII line intensities are $2.26 \pm 0.6$ photons $\mathrm{s}^{-1} \mathrm{~cm}^{-2} \mathrm{sr}^{-1}$ (line units, LU, from now on) and $0.56 \pm 0.48 \mathrm{LU}$ for MBM20, and $5.68 \pm 1.04 \mathrm{LU}$ and $1.32 \pm 0.79 \mathrm{LU}$ for the $\mathrm{EH}$, respectively. Following the same recipe used in Galeazzi et al. (2007), we can evaluate $\mathrm{O}$ VII and $\mathrm{O}$ VIII emission of the foreground $(\mathrm{LB}+\mathrm{SWCX})$ and background $(\mathrm{GH})$ components. Using the expression for cross-section per hydrogen atom for a cosmic abundance plasma derived by Morrison \& McCammon (1983), we find that MBM20 absorbs about $75 \%$ of the background O VII emission and about $61 \%$ of the background O VIII emission, while the $\mathrm{EH}$ absorbs about $8 \%$ of the background $\mathrm{O}$ VII emission and about $5 \%$ of the background $\mathrm{O}$ VIII emission. Combining these data with the result of our observations we obtain, for O VII and O VIII, respectively, $0.99 \pm 0.91 \mathrm{LU}$ and $0.014 \pm 1.01 \mathrm{LU}$ for the foreground and 5.10 $\pm 1.79 \mathrm{LU}$ and $1.42 \pm 1.74 \mathrm{LU}$ for the background.

We evaluated the electron density and thermal pressure of the $\mathrm{GH}$ and the LB, using the same procedure discussed in Galeazzi et al. (2007). Assuming the foreground component is due solely to LB emission, we obtain lower and upper limits for the plasma density of 0.015 and $0.018 \mathrm{~cm}^{-3} \mathrm{~K}$ and limits of 23,500 and

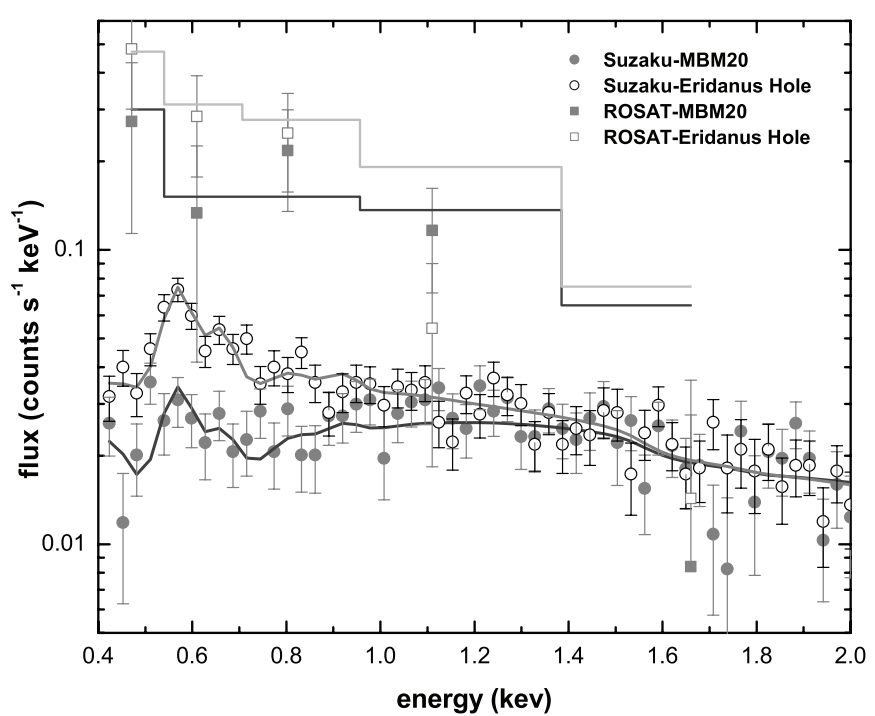

Figure 5. Global fits for MBM20 (dark gray) and EH (gray) using data from our Suzaku observations (circles) and RASS (squares).

$28,800 \mathrm{~cm}^{-3} \mathrm{~K}$ for the plasma pressure. Similarly, assuming that the absorbed plasma component is due solely to $\mathrm{GH}$ emission, we obtain a plasma density ranging from 0.0005 to $0.0014 \mathrm{~cm}^{-3}$ and a pressure between $3.3 \times 10^{3}$ and $5.8 \times 10^{3} \mathrm{~cm}^{-3} \mathrm{~K}$.

We also used the non-equilibrium plasma model GNEI (Borkowski et al. 2001), a non-equilibrium model characterized by a constant postshock electron temperature and by its ionization age, to fit our data. While we obtained a good fit, similar to that shown in Figure 5, and an electron density in the range $0.013-0.158 \mathrm{~cm}^{-3}$, we derived a value for the age of the LB of $\leqslant 0.9 \mathrm{Myr}$, which is quite small in comparison with generally accepted models (e.g., Edgar \& Cox 1993).

\section{COMPARING SUZAKU AND XMM-NEWTON OBSERVATIONS OF THE SOFT X-RAY BACKGROUND}

The temperature and emission measures we obtained from the Suzaku data are significantly different from those determined from the XMM-Newton analysis in the same pointing directions. For a visual estimate of the difference, we folded our Suzaku model through the XMM-Newton response and compared it with the XMM-Newton spectra (see Figure 6). The difference in these spectra would be consistent with a time dependent component of the foreground emission, attributable to SWCX, which we will discuss in detail in the next few sections. The excess is clearly significant in both data sets.

So far only a few targets with the proper characteristics for shadow experiments have been observed with any of the three major X-ray satellites (Chandra, XMM-Newton, and Suzaku). In addition to the MBM20 observations discussed, we point out the observations of the neutral hydrogen cloud MBM12 performed with Chandra (Smith et al. 2005) and Suzaku (Smith et al. 2006) and that of a relatively dense neutral hydrogen filament in the southern galactic hemisphere (Henley \& Shelton 2008).

Table 3 summarizes the O VII and O VIII flux for all the available observations. Data from McCammon et al. (2002) are also reported for comparison. In McCammon et al. a highresolution measurement over a 1 sr field of view near the north Galactic pole was performed using cryogenic microcalorimeters mounted on a sounding rocket. Tables 4 and 5 give a summary dividing the results in foreground and background emission. Where a fit with a plasma model has been performed, the best 

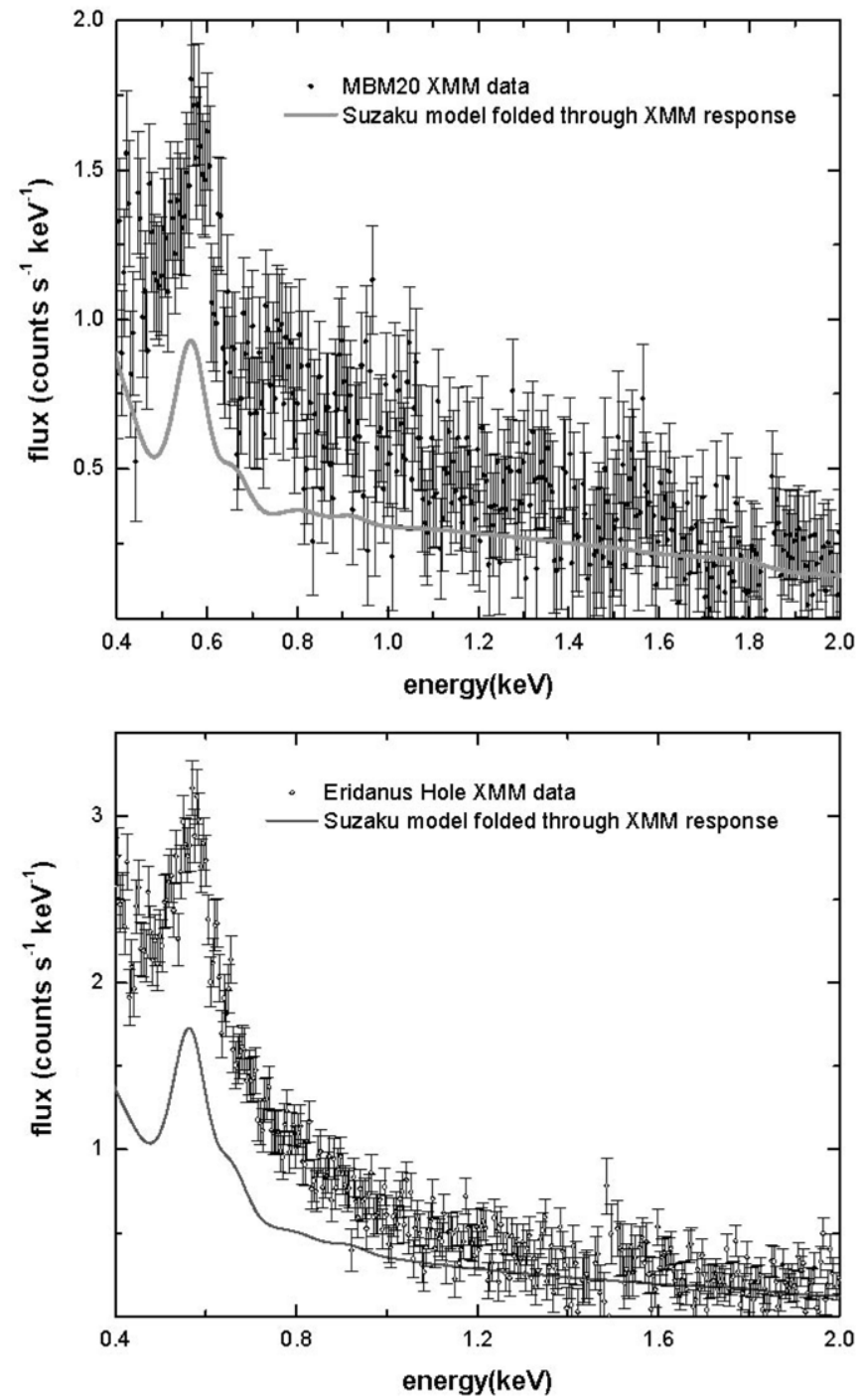

Figure 6. Top: comparison between XMM-Newton MBM20 spectra and Suzaku model folded through XMM-Newton response. Bottom: same as top for EH.

fit parameters for temperature and emission measure are also reported.

While the amount of available data is limited, we identified a few general trends that we want to point out.

1. Each target has been observed at least twice in the past 8 years, but the results from multiple observations of the same target do not agree, at various levels, with each other. This is evidence of a significant contribution from SWCX, the only component of the diffuse X-ray background that should change with time on such a short timescale. Moreover, when we separate foreground and background oxygen line emission, the component that changes with time seems to be the foreground one, while the background does not change, within the errors, between different observations of the same target, strengthening the notion that the variation is due to SWCX. We want to point out, however, that multiple observations of the same target have been performed with different satellites, i.e., different data reduction analysis, background subtraction schemes, etc., which have different systematic uncertainties.

2. The change in oxygen line emission between different observations of the same target can be used to estimate the typ-
Table 3

Summary of the Oxygen Line Emission for MBM12, MBM20, and the Filament in the Southern Galactic Hemisphere (SGF)

\begin{tabular}{|c|c|c|c|}
\hline Experiment & $\mathrm{NH}\left(10^{20} \mathrm{~cm}^{-2}\right)$ & O VII & O VIII \\
\hline MBM20 & 15.9 & & \\
\hline XMM-Newton & & $3.89 \pm 0.56$ & $0.68 \pm 0.24$ \\
\hline Suzaku & & $2.26 \pm 0.60$ & $0.56 \pm 0.48$ \\
\hline Eridanus Hole & 0.0086 & & \\
\hline XMM-Newton & & $7.26 \pm 0.34$ & $1.63 \pm 0.17$ \\
\hline Suzaku & & $5.68 \pm 1.04$ & $1.32 \pm 0.79$ \\
\hline MBM12-on cloud & 40 & & \\
\hline Chandra & & $1.79 \pm 0.55$ & $2.34 \pm 0.36$ \\
\hline Suzaku & & $3.34 \pm 0.26$ & $0.24 \pm 0.10$ \\
\hline MBM12-off cloud & 8.7 & & \\
\hline Suzaku & & $5.68 \pm 0.42$ & $0.01 \pm 0.19$ \\
\hline Henley et al. on filament & 9.6 & & \\
\hline$X M M-N e w t o n$ & & $10.65 \pm 0.80$ & $3.91 \pm 0.26$ \\
\hline Suzaku & & $6.51 \pm 0.41$ & $2.54 \pm 0.26$ \\
\hline Henley et al. off filament & 1.9 & & \\
\hline XMM-Newton & & $13.86 \pm 1.44$ & $2.81 \pm 0.59$ \\
\hline Suzaku & & $10.53 \pm 0.61$ & $3.21 \pm 0.31$ \\
\hline McCammon et al. (2002) & 1.8 & & \\
\hline XQC & & $4.8 \pm 0.8$ & $1.6 \pm 0.4$ \\
\hline
\end{tabular}

Note. The data from McCammon et al. (2002) are also reported.

Table 4

Summary of the Foreground Emission for the Targets Discussed in this Section

\begin{tabular}{lcccc}
\hline \hline Experiment & $\begin{array}{c}T \\
\left(10^{6} \mathrm{~K}\right)\end{array}$ & $\begin{array}{c}\mathrm{EM} \\
\left(\mathrm{cm}^{-6} \mathrm{pc}\right)\end{array}$ & $\begin{array}{c}\text { O vII } \\
(\mathrm{LU})\end{array}$ & $\begin{array}{c}\text { O VIII } \\
(\mathrm{LU})\end{array}$ \\
\hline MBM20 & & & & \\
XMM & 1.12 & 0.0088 & $2.63 \pm 0.78$ & $0.03 \pm 0.43$ \\
Suzaku & 0.70 & 0.097 & $0.99 \pm 0.91$ & $0.01 \pm 1.01$ \\
MBM12 & & & & \\
Suzaku & $\sim 1.2$ & & $3.34 \pm 0.26$ & $0.24 \pm 0.1$ \\
Chandra & & & $1.79 \pm 0.55$ & $2.34 \pm 0.36$ \\
SGF & & & & $1.0 \pm 1.1$ \\
Suzaku & 0.95 & 0.0064 & $1.1 \pm 1.1$ & $<1$ \\
XMM & 1.15 & 0.018 & $6.2 \pm 2.8$ & $<1$ \\
\hline
\end{tabular}

Table 5

Summary of the Background Emission for the Targets Discussed in this Section

\begin{tabular}{lcccc}
\hline \hline Experiment & $\begin{array}{c}T \\
\left(10^{6} \mathrm{~K}\right)\end{array}$ & $\begin{array}{c}\text { EM } \\
\left(\mathrm{cm}^{-6} \mathrm{pc}\right)\end{array}$ & $\begin{array}{c}\text { O vII } \\
(\mathrm{LU})\end{array}$ & $\begin{array}{c}\text { O VIII } \\
(\mathrm{LU})\end{array}$ \\
\hline MBM20 & & & & \\
XMM & 2.23 & 0.0034 & $5.03 \pm 0.98$ & $1.68 \pm 0.53$ \\
Suzaku & 2.15 & 0.0031 & $5.10 \pm 1.79$ & $1.42 \pm 1.74$ \\
MBM12 & & & $2.34 \pm 0.33$ & $0.77 \pm 0.16$ \\
Suzaku & & & & \\
SGF & & & & \\
Suzaku & $1.29 / 3.16$ & $0.034 / 0.0065$ & $8.8 \pm 4.9$ & $2.4 \pm 1.5$ \\
XMM & $0.85 / 2.69$ & $0.17 / 0.011$ & $10.9 \pm 2$ & \\
\hline
\end{tabular}

ical flux variation of the SWCX emission. The O vII emission varies between $1.55 \pm 0.61 \mathrm{LU}$ and $4.14 \pm 0.90 \mathrm{LU}$ while the $\mathrm{O}$ VIII emission varies between $0.22 \pm 0.72 \mathrm{LU}$ and $2.10 \pm 0.37 \mathrm{LU}$. The detailed results are reported in Table 6.

3. High-resolution investigations of the diffuse X-ray emission have shown that a simple one-temperature plasma in equilibrium cannot explain the observed spectra (McCammon et al. 2002; Sanders et al. 2001). However, while CCD detectors are a significant step forward from proportional counters, their resolution is still quite limited 
Table 6

O vII and O vIII Variations Between Multiple Observations of the Same Object

\begin{tabular}{lcc}
\hline \multicolumn{1}{c}{ Target } & $\Delta[\mathrm{O}$ vII $](\mathrm{LU})$ & $\Delta[\mathrm{O}$ VIII $](\mathrm{LU})$ \\
\hline MBM20 & $1.63 \pm 0.82$ & $0.12 \pm 0.54$ \\
Eridanus Hole & $1.58 \pm 1.09$ & $0.31 \pm 1.33$ \\
MBM12 & $1.55 \pm 0.61$ & $2.10 \pm 0.37$ \\
SGF On-filament & $4.14 \pm 0.90$ & $1.37 \pm 0.37$ \\
SGF Off-filament & $3.33 \pm 1.56$ & $0.40 \pm 0.67$ \\
\hline
\end{tabular}

and insufficient to investigate the issue. Equilibrium plasma models seem to be still sufficient to fit the spectra and, while there have been attempts at using more sophisticated models, it is impossible to distinguish between them. At this point the available data are adequately fit with a plasma thermal emission from the LB, with temperature around 1 million deg, and either one or two temperature thermal plasma components for the $\mathrm{GH}$, with temperatures between 2 and 3 million deg.

4. Except for the Chandra observation of MBM12, with its very unusual $\mathrm{O}$ vIII emission, all other observations seem to indicate that the foreground $\mathrm{O}$ VIII emission is either very small or compatible with 0 . Typical LB models do not predict significant $\mathrm{O}$ VIII emission and this seems to indicate that the SWCX component does not normally have any significant emission in $\mathrm{O}$ VIII either.

5. When the assumption is made that all the foreground emission is due to LB emission, the derived values for the plasma temperature, density, and pressure seem to be in good agreement with the predictions from the most commonly accepted models of the origin and structure of the LB (e.g., Smith \& Cox 2001).

\section{SWCX MODEL TO DATA COMPARISON}

The heliospheric SWCX model we use for our simulations is extensively described in Koutroumpa et al. (2006, 2007). This model is a self-consistent calculation of the SWCX X-ray line emission for any LOS through the heliosphere and for any observation date, based on three-dimensional grids of the interstellar (IS) neutral species ( $\mathrm{H}$ and $\mathrm{He}$ ) distributions in the heliosphere modulated by solar activity conditions (gravity, radiation pressure, and ionization processes which are anisotropic due to the latitudinal anisotropy of the solar wind mass flux and solar radiation). Highly charged heavy solar wind (SW) ions are propagated radially through these grids and the charge-transfer collision rates are calculated for each of the ion species, including the evolution of their density due to charge-transfer with the IS atoms. With this process, we establish three-dimensional emissivity grids for each SW ion species, using photon emission yields computed by Kharchenko \& Dalgarno (2000) for each spectral line following charge exchange with the corresponding neutral species ( $\mathrm{H}$ and $\mathrm{He}$ individually). Finally, the $\mathrm{X}$-ray line emission is integrated along any LOS and observation geometry (for each observation date) in order to build the complete spectrum of SWCX emission in the given direction. For comparison to present $\mathrm{X}$-ray observations we use the $\mathrm{O}$ VII triplet at $0.57 \mathrm{keV}$ and the $\mathrm{O}$ VIII line at $0.65 \mathrm{keV}$, as they are the strongest spectral features and provide the best signal-to-noise ratio for the observations.

We have conducted simulations for each of the MBM20 and $\mathrm{EH}$ observations accounting as close as possible for average solar activity conditions corresponding to the observation period. Solar activity is reflected both in the IS neutral distribu- tions (by means of ionization rates that are increased and less anisotropic in solar maximum), and in the solar wind ionic composition (abundances and charge state distributions) and spatial distribution.

Details of the solar activity effect on the neutral $\mathrm{H}$ and $\mathrm{He}$ distributions, along with the latitude-dependant ionization rates used in the model for maximum (e.g., 2001), intermediate (e.g., 2003-2004), and minimum (e.g., 2007-2008) solar conditions are given in Koutroumpa et al. (2009).

The latitude dependence of the solar wind also affects the highly charged heavy ion distribution, where abundances depend on the solar wind type. During minimum solar activity, the solar wind is considered to be highly anisotropic, with a narrow equatorial zone (within $\pm 20^{\circ}$ of the solar equatorial plane) of slow solar wind with an average speed of $\sim 400 \mathrm{~km}$ $\mathrm{s}^{-1}$ and the fast solar wind emitted from the polar coronal holes at a speed of $\sim 700 \mathrm{~km} \mathrm{~s}^{-1}$. The slow solar wind has a proton density of $\sim 6.5 \mathrm{~cm}^{3}$ at $1 \mathrm{AU}$, while the fast flow is less dense at $\sim 3.2 \mathrm{~cm}^{3}$ at $1 \mathrm{AU}$. At solar maximum, the solar wind spatial distribution is considered to be a complex mix of slow and fast wind states that is in general approximated with an average slow wind flux. The ionic composition of the two flows can be very different with the average oxygen content varying from $[\mathrm{O} / \mathrm{H}]=1 / 1780$ in the slow wind and $[\mathrm{O} / \mathrm{H}]=$ $1 / 1550$ in the fast flow. The charge-state distributions change as well, with the higher charge-states strongly depleted (or even completely absent, as for example $\mathrm{O}^{+8}$ in the fast solar wind. For our model we adopt the oxygen relative abundances published in Schwadron \& Cravens $(2000):\left(\mathrm{O}^{+7}, \mathrm{O}^{+8}\right)=(0.2,0.07)$ for the slow wind and $\left(\mathrm{O}^{+7}, \mathrm{O}^{+8}\right)=(0.07,0.0)$ for the fast wind, based on data from the Ulysses SWICS instrument.

The XMM-Newton observations of MBM20 and the EH were performed during 2004, which corresponds to intermediate solar conditions, while for the Suzaku observations, performed in 2007-2008, solar minimum conditions are most appropriate. The main difference in the SW heavy ion distribution between the two periods (two sets of coupled observations) is the spatial (latitudinal) distribution of the slow and fast solar wind flows. For the Suzaku simulations (solar minimum) the slow SW is expanding in interplanetary space through a $\pm 20^{\circ}$ equatorial zone on the solar surface, while the fast SW flow occupies the rest of the space. For the intermediate 2003-2004 period (XMMNewton observations) we assume that there is no fast wind flow in interplanetary space (same approach as for solar maximum), in order to estimate the quiescent (outside potential coronal mass ejection or solar flare) upper limit for the resulting SWCX $\mathrm{X}$-ray emission. Indeed, as demonstrated in Koutroumpa et al. (2006, 2007), for high ecliptic-latitude LOS, as is the case for the MBM20 and EH observations (decl. $\sim-38^{\circ}$ ), the oxygen line intensity decreases from solar maximum to solar minimum conditions as the LOS crosses larger fast wind regions where the parent ions are strongly depleted.

In Table 7, we summarize the SWCX model results for the oxygen line intensities for the four observations. As expected, model A, which assumes average solar wind conditions as described above, predicts a significant decrease in the SWCX oxygen line intensities as we progress from near solar maximum (XMM-Newton) to solar minimum (Suzaku), since we are observing at high southern ecliptic latitudes. Also, the model predicts a decrease in the heliospheric SWCX emission when shifting the view direction from the EH (off-cloud) to the MBM20 (on-cloud) direction. This decrease is of the order of $8 \%$ for the XMM-Newton observations and of the order of 

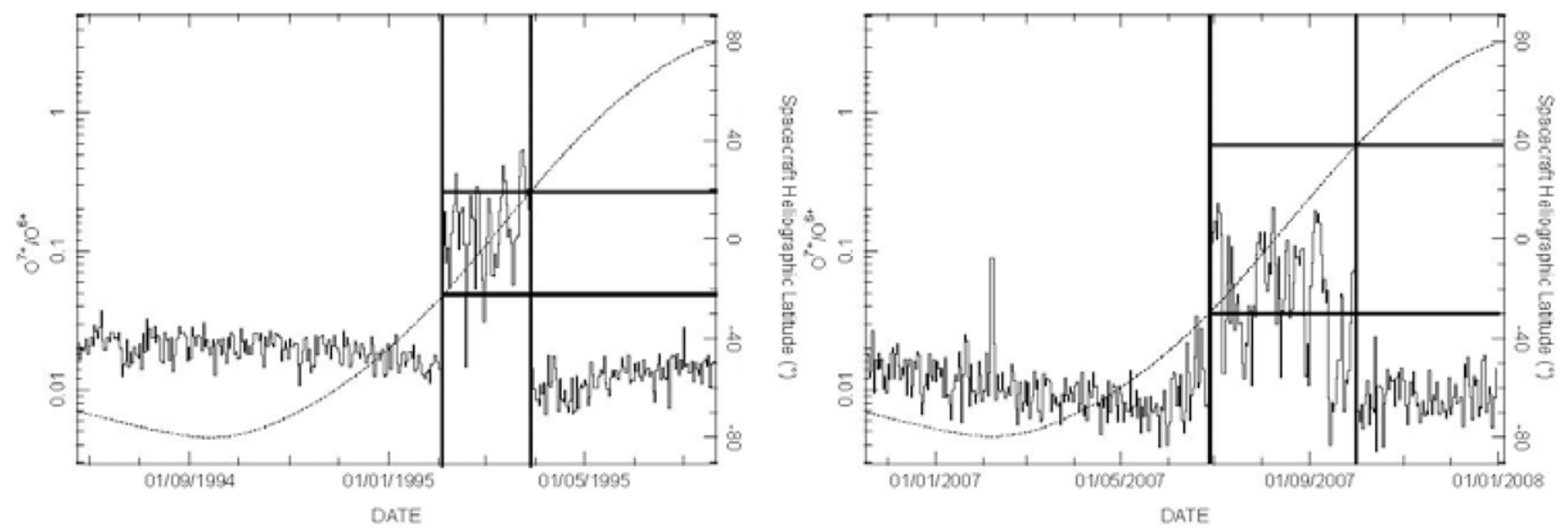

Figure 7. Left: Ulysses $/$ SWICS $\mathrm{O}^{+7} / \mathrm{O}^{+6}$ ratio data (plain) during the 1995 crossing of the solar equatorial plane. The dotted curve marks the spacecraft heliographic latitude on the right axis. The vertical and horizontal plain lines denote the limits of the slow solar wind equatorial zone in terms of crossing time and heliographic latitude, respectively. Right: same as left, except for the 2007 crossing of the solar equatorial plane.

Table 7

Model SWCX Oxygen Line Intensities in LU

\begin{tabular}{|c|c|c|c|c|c|c|c|}
\hline \multirow[t]{2}{*}{ ObsId } & \multirow[t]{2}{*}{ Target } & \multicolumn{2}{|c|}{ Model A } & \multicolumn{2}{|c|}{ Model Ba } & \multicolumn{2}{|c|}{ Model B $1^{\mathrm{b}}$} \\
\hline & & O VII & O VIII & O VII & O VIII & O VII & O VIII \\
\hline 0203900101 & $\mathrm{EH}$ & 2.04 & 0.80 & 2.04 & 0.80 & 2.04 & 0.80 \\
\hline 0203900201 & MBM20 & 1.88 & 0.74 & 1.88 & 0.74 & 1.88 & 0.74 \\
\hline 502076010 & $\mathrm{EH}$ & 1.14 & 0.28 & 1.45 & 0.42 & 0.29 & 0.42 \\
\hline 502075010 & MBM20 & 0.81 & 0.16 & 1.29 & 0.38 & 0.15 & 0.38 \\
\hline
\end{tabular}

Notes.

a A larger latitudinal extent $\left( \pm 30^{\circ}\right)$ of slow wind heavy ion abundances is assumed for solar minimum (Suzaku). XMM-Newton simulation assumptions remain unchanged.

b A real-time $\mathrm{O}^{+7}$ measured density is applied to model $\mathrm{B}$ simulation for the Suzaku observations. $\mathrm{O}^{+7}$ data taken in situ at the $\mathrm{L} 1$ point are extrapolated to the whole LOS. XMM-Newton $\mathrm{O}$ vII simulations and $\mathrm{O}$ vIII simulations remain unchanged.

$30 \%$ up to $45 \%$ (for O viI) for the Suzaku observations. Such a large difference can be explained by the large interval separating the two Suzaku observations combined with the inclination of the equatorial SW zone with respect to the ecliptic plane (due to the 7.25 inclination of the solar axis with respect to the ecliptic axis). Indeed, the Suzaku observations of MBM20 and EH were performed at an observed ecliptic longitude of $142^{\circ}$ and $306^{\circ}$, respectively, separated by six months, while the LOS was pointing at $\sim 37^{\circ}$ south. Since the solar equator ascending node is $\Omega=73.67$, the EH LOS was looking through a larger region of the oxygen-rich slow solar wind equatorial zone than the MBM20 LOS.
One step to further improve the accuracy of our prediction is to apply reasonable assumptions to the SWCX simulations. First, evidence from the Ulysses/SWICS $\mathrm{O}^{+7} / \mathrm{O}^{+6}$ ratio data (which is a proxy for the flow speed/type) during the 2007 (minimum) crossing of the equatorial slow wind zone (Figure 7) shows that this latter was in fact more extended in latitude (more than $\pm 30^{\circ}$ ) than during the previous solar minimum (1996) that served as a reference for the minimum SW conditions applied in the SWCX model. In order to investigate the effect of such a possibility we performed a second simulation of the Suzaku EH/MBM20 observations introducing a $\pm 30^{\circ}$ slow SW zone as input. The results are also noted in Table 7 (Model B). However, in situ measurements with $A C E$ at the L1 point show unusually low $\mathrm{O}^{+7}$ abundances for the Suzaku observations period, almost an order of magnitude lower than the average slow wind conditions (11.5\% during the MBM20 observation and 20\% during the $\mathrm{EH}$ one). For solar maximum (XMM-Newton observations), the $\mathrm{O}^{+7}$ abundance in the $A C E$ data does not show significant deviation from average slow wind values. $\mathrm{O}^{+8}$ measurements are too sparse to allow a significant quantitative analysis of the data, and therefore we will make no assumption for these data. If we apply $11.5 \%$ and $20 \%$ correction factors to the Suzaku's MBM20 and EH O VII line intensities predicted from Model B, we obtain the values noted as Model B1 in Table 7.

To compare the model results to the observations of MBM20 and the EH we must consider that MBM 20 absorbs about $75 \%$ of the background $\mathrm{O}$ VII emission and about $61 \%$ of the background $\mathrm{O}$ VIII emission and therefore we expect a significant contamination from the background emission. Table 8 summarizes the final values predicted in the SWCX simulations (model B1 from Table 7), along with the measured O vII and O VII fluxes, the

Table 8

Data and SWCX Model Oxygen Line Intensities in LU

\begin{tabular}{|c|c|c|c|c|c|c|c|}
\hline Mission & Target & \multicolumn{2}{|c|}{ Data } & \multicolumn{2}{|c|}{ Model B1 } & \multicolumn{2}{|c|}{ Residual } \\
\hline & $\mathrm{EH}$ & $7.37 \pm 0.34$ & $1.73 \pm 0.17$ & 2.04 & 0.80 & $5.23 \pm 0.34$ & $0.83 \pm 0.17$ \\
\hline$X M M$ & MBM20 & $3.59 \pm 0.56$ & $0.72 \pm 0.24$ & 1.88 & 0.74 & $2.00 \pm 0.56$ & $\sim 0$ \\
\hline & Foreground & $2.63 \pm 0.78$ & $0.03 \pm 0.43$ & 1.96 & 0.77 & $0.67 \pm 0.78$ & $\sim 0$ \\
\hline & $\mathrm{EH}$ & $6.68 \pm 1.04$ & $1.32 \pm 0.79$ & 0.29 & 0.42 & $5.39 \pm 1.04$ & $0.9 \pm 0.79$ \\
\hline
\end{tabular}

Note. The foreground values are from Table 4 for the data and are the average of the EH and MBM20 values for the models. 
estimated foreground (local) flux from Table 4, and the predicted residual cosmic background (data minus model).

As the results in Table 8 show, the SWCX O VII prediction is comparable, within $1 \sigma$, with the measured local emission. Also, the residual $\mathrm{O}$ vII cosmic background has a constant value, within error bars, for all on-cloud and off-cloud observations that is consistent with the extrapolated background emission reported in Table 5. Both results seem to indicate that the O VII foreground emission is dominated by SWCX. This conclusion is also supported by a previous application of the model to the MBM12 observations (Koutroumpa et al. 2007). Due to the significantly higher absorption of MBM12, the model results were compared directly to the total measured flux and the agreement was within $30 \%$. We point out that this conclusion does not preclude the existence or a Local Hot Bubble which is expected to emit X-rays primarily at lower energy, in the $1 / 4 \mathrm{keV}$ band. Models predict a LB O vII surface brightness of about 0.25 LU.

The $\mathrm{O}$ VIII results are not as clear, as the measured data are consistent with a zero local emission, while the model predicts a small, but non-zero emission. However, as mentioned before, the $A C E \mathrm{O}^{8+}$ data are too sparse and could not be used as input for our model. The negligible $\mathrm{O}$ VIII flux could therefore simply be caused by a smaller than expected $\mathrm{O}^{8+}$ density in the solar wind.

\section{CONCLUSION}

We used Suzaku observations of the molecular cloud MBM20 and a low neutral hydrogen column density region nearby to separate and characterize the foreground and background diffuse X-ray emission. We measured a foreground flux of $0.99 \pm 0.91 \mathrm{LU}$ and $0.01 \pm 1.01 \mathrm{LU}$ for $\mathrm{O}$ VII and O VIII, respectively, and a background flux of $5.10 \pm 1.79 \mathrm{LU}$ and $1.42 \pm 1.74$ LU of O VII and O VIII, respectively.

The comparison with a previous observation of the same regions with $X M M$-Newton indicates a significant change in the foreground flux which we attribute to SWCX. By combining our results with similar multiple shadow investigation of the same target we find that the O VII emission varies between $1.55 \pm$ $0.61 \mathrm{LU}$ and $4.14 \pm 0.90 \mathrm{LU}$ between multiple observations of the same target. The $\mathrm{O}$ vIII emission, except for a single case with a change of $2.10 \pm 0.37 \mathrm{LU}$, is generally compatible with zero, possibly indicating a very low density of $\mathrm{O}^{8+}$ in the solar wind.

We also compared our results with a SWCX model to constrain its $\mathrm{O}$ VII and $\mathrm{O}$ VIII emission. The model is in good agreement with the measured $\mathrm{O}$ vII flux and seems to indicate that most of the O VII foreground emission is due to SWCX. This is not necessarily inconsistent with the existence of a local hot bubble which is expected to emit predominantly at lower energy, in the $1 / 4 \mathrm{keV}$ band.

With the limited energy resolution of the CCD detectors, the foreground emission can also be modeled with an unabsorbed plasma model and the background one with a one temperature absorbed plasma model plus an absorbed power law, as done in previous papers. A global fit using both data sets and RASS Data for the same targets is consistent with a foreground plasma emission with $T=0.7 \times 10^{6} \mathrm{~K}$ and $\mathrm{EM}=0.096 \mathrm{~cm}^{-6} \mathrm{pc}$ and a background plasma emission with $T=2.15 \times 10^{6} \mathrm{~K}$ and $\mathrm{EM}=0.0031 \mathrm{~cm}^{-6} \mathrm{pc}$. We also obtained a good fit by using a non-equilibrium plasma model for the foreground emission, however, the inferred age of the plasma is $\leqslant 0.9 \mathrm{Myr}$, inconsistent with any LB model.

\section{REFERENCES}

Anders, E., \& Ebihara, 1982, Geochim. Cosmochim. Acta, 46, 2363

Arnaud, K., \& Dormer, B. 2002, XSPEC: An X-Ray Spectral Fitting package, User's Guide for version 11.2.x, HEASARC, Exploration of the Universe Division (Greenbelt, MD: NASA/GSFC), http://heasarc.gsfc.nasa.gov/ docs/xanadu/xspec/manual/manual.html

Borkowski, K. J., Lyerly, W. J., \& Reynolds, S. P. 2001, ApJ, 548, 820

Boulanger, F., \& Perault, M. 1988, ApJ, 330, 964

Edgar, R. J., \& Cox, D. P. 1993, ApJ, 413, 190

Galeazzi, M., Gupta, A., Covey, K., \& Ursino, E. 2007, ApJ, 658, 1081

Galeazzi, M., Gupta, A., \& Ursino, E. 2009, ApJ, 695, 1127

Gupta, A., \& Galeazzi, M. 2009, ApJ, 702, 270

Hearty, T., et al. 2000, A\&A, 357, 681

Henley, D. B., \& Shelton, R. L. 2008, ApJ, 675, 335

Henley, D. B., Shelton, R. L., \& Kuntz, K. D. 2007, ApJ, 661, 304

Ishisaki, Y., et al. 2007, PASJ, 59, 113

Kharchenko, V., \& Dalgarno, A. 2000, J. Geophys. Res., 105, 18351

Koutroumpa, D., et al. 2006, A\&A, 460, 289

Koutroumpa, D., et al. 2007, A\&A, 475, 901

Koutroumpa, D., et al. 2009, ApJ, 697, 1214

Koyama, K., et al. 2007, PASJ, 59, s23

McCammon, D., et al. 2002, ApJ, 576, 188

Mitsuda, K., et al. 2007, PASJ, 59s, 1

Morrison, R., \& McCammon, D. 1983, ApJ, 270, 119

Sanders, W. T., et al. 2001, ApJ, 554, 694

Schwadron, N. A., \& Cravens, T. E. 2000, ApJ, 544, 558

Smith, R. K., \& Cox, D. P. 2001, ApJS, 134, 283

Smith, R. K., et al. 2005, ApJ, 623, 225

Smith, R. K., et al. 2006, PASJ, 59, 141

Snowden, S. L., et al. 1998, ApJ, 493, 715

Tawa, N., et al. 2008, PASJ, 60s, 11 